\title{
Glossary
}

\section{Basic concepts in population health and health care}

\author{
B Starfield
}

These definitions are intended to be operational definitions; that is, they are designed to facilitate the development of specific measures to assess their attainment, on the assumption that it does no good to provide a definition if it does not facilitate assessment of change or the extent to which a goal has been reached. For example, most definitions of equity use the terms "fairness" or "just", but these characteristics seem to defy measurement. This is why the definitions may be quite different from those you are accustomed to seeing or have encountered.

The concepts defined herein are ones that either have no well accepted definitions or have definitions that have not proved useful either in informing my own research or in interpreting the literature. They derive from my experiences in designing research and trying to glean useful ideas from a diverse set of readings. Although the definitions are not likely to be readily accepted by everyone who considers them, the intent is to generate discussion so that research may move ahead with better conceptualisation of research topics and variables and more consistent interpretation of research findings.

\section{Access and use}

(1) Accessibility (of health services): aspects of the structure of health services or health facilities that enhance the ability of people to reach a health care practitioner, in terms of location, time, and ease of approach.

(2) Access (to health services): the perceptions and experiences of people as to their ease in reaching health services or health facilities in terms of location, time, and ease of approach.

(3) Utilisation/use (of health services): experience of people as to their receipt of health care services of different types.

Chronic disease, illness, or condition: a disease, illness, or condition that increases the risk of long term disability, handicap, or death and is unlikely to remit, although the occurrence and/or severity of its manifestations may vary over time.

The distinction between a chronic illness and a non-chronic illness is generally made according to the likelihood of its lasting for 12 months or more and resulting in functional limitations and/or need for ongoing medical care. ${ }^{1}$ Data from population-based studies of visits to medical facilities show that people with a "diagnosed" chronic illness often do not have that diagnosis from year to year, and people who have one or more of a large number of non-chronic illnesses that are widely believed to be short-term often have recurring episodes of the same or related illnesses over the long term. The absence of a clear distinction between chronic illness and non-chronic illness is responsible for low agreement in lists of the two types of conditions among different researchers and analysts.

Community: a unit, generally geographically defined, which is the locus of basic political and social responsibility and in which everyday social interactions involving all or most of the spectrum of life activities of the people within it takes place.

Determinants of health: the wide variety of interacting proximate and distal influences on the health of individuals and populations, including but not limited to political contexts, policies, distribution of power and wealth, social and physical and social environments, health systems and services, as well as genetic, biological, and historico-cultural characteristics (fig 1).

The use of the term "determinants" rather than "determinant" is intentional. With the exception of Mendelian dominant disorders (and, even here, their severity might be modifiable by the presence of other factors), there is no single determinant of disease or illness.

\section{Episodes}

Episodes of illness: clusters of visits and/or procedures that are related to a particular illness, disease, or condition.

\section{Equity}

Equity in health: the absence of systematic differences in one or more aspects of health status across socially, demographically, or geographically defined population groups.

Inequity in health: the presence of systematic differences in one or more aspects of health across socially, demographically, or geographically defined population groups.

Inequity in access to health services: differences in access to health services for equal health needs and/or absence of enhanced access for 


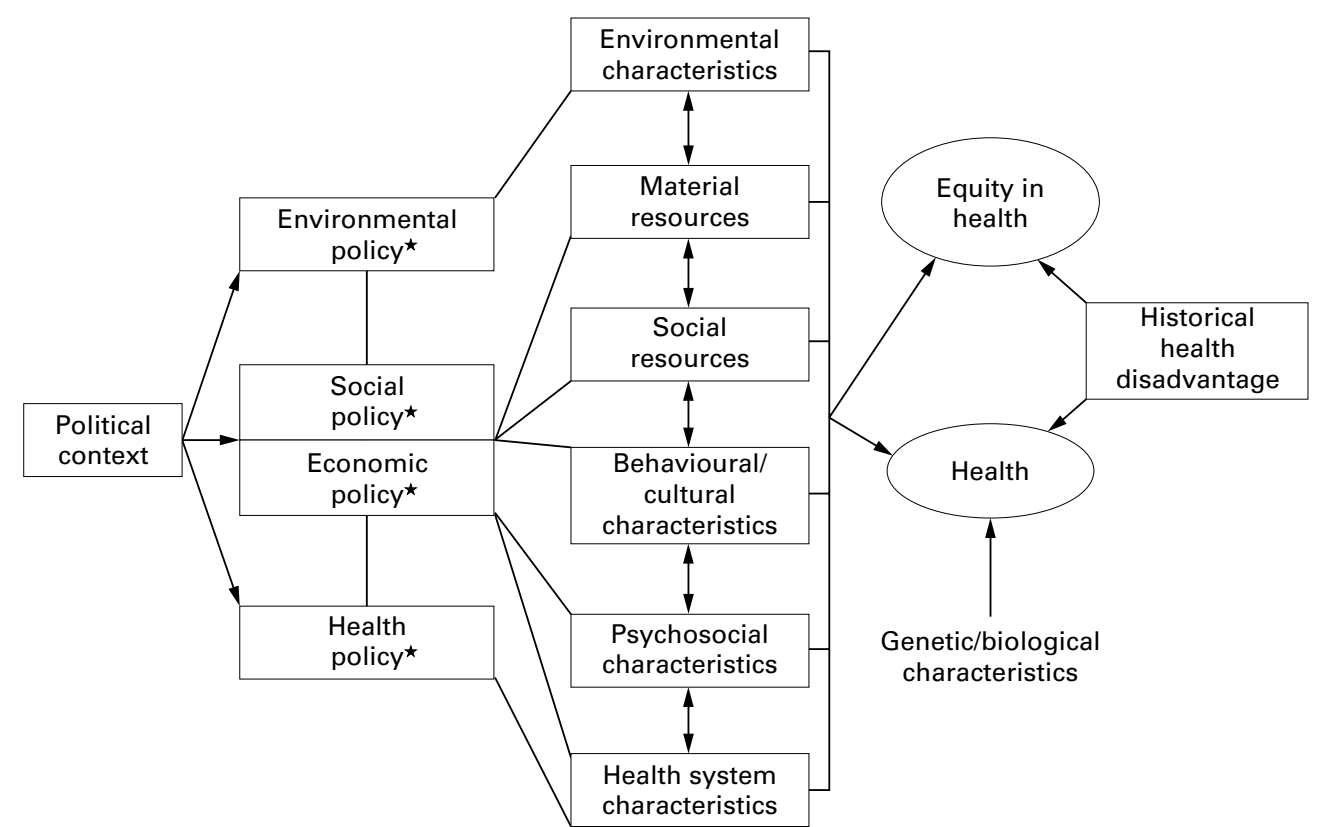

Figure 1 A conceptual framework of health determinants: population model. *Policy reflects a country's approach to the distribution of power (political jurisdiction). Determinants of health at the individual level are the same but without "Equity in health outcomes."

socially, demographically, or geographically defined population groups with greater health needs.

Inequity in receipt of health services: inequalities in the receipt of health services for equal health needs and/or failure to receive increased or augmented services for socially, demographically, or geographically defined population groups with greater health needs.

Functional status: the capacity to engage in activities of daily living and social role activities. (Increasingly, functional status is considered to represent the concept of "health" in its behavioural manifestations. However, health is a broader concept, as noted below.)

Guidelines: recommendations or suggestions for organising or delivering services of various types. Guidelines are distinguished from regulations by their voluntary nature, but adherence is generally facilitated by professional expectations rather than formal requirements. Guidelines for public health activities, such as those for laboratory standards, are distinguished from clinical guidelines, which describe interventions that are indicated (or not indicated) in the diagnosis and management of specific diseases or conditions. Increasingly, the development of clinical guidelines is informed by scientific evidence of the benefit of the recommended procedures, thus giving rise to the field known as "evidence-based medicine". The development of administrative guidelines is more often based on evidence from practice of systematically poor quality when certain structural elements of health systems are not appropriately or adequately maintained.

Health: the extent to which an individual or group is able, on the one hand, to realise aspirations and satisfy needs and, on the other hand, to cope with the interpersonal, social, biological, and physical environments. Health is therefore a resource for everyday life, not the objective of living; it is a positive concept embracing social and personal resources as well as physical and psychological capacities.

Health can be represented, operationally, on the individual or population level.

On the individual level, health (in contrast with disease or mortality rates) is either the sum of many separate indicators weighted in an appropriate way, or as a summary measure that reflects the manifestations of the individual indicators. There is as yet no agreed upon way to weight the various individual indicators. Summary measures such as years of potential life lost or disability adjusted life years (DALYS), or DALES (disability adjusted life expectancies), may be reasonable approximations, although their limitations have been recognised.

Population health is not merely the sum of the health of individuals; it also entails consideration of the nature of the distribution of health throughout the population.

The first part of the definition of health, slightly adapted, was proposed in a draft document by WHO-EURO in the early 1980s. It was subsequently adopted, at least in part, by the Ottawa Declaration in $1987 .^{2}$

\section{Health promotion and protection}

(1) Health promotion: activities, usually directed at individuals, to maximise the development of resilience to a threat or threats to health. (Some academicians use the term "health promotion" to designate activities that involve consideration of political and social determinants; this conceptualisation is included under "Determinants of Health" in this glossary.) 
(2) Health protection: activities undertaken to reduce the likelihood of occurrence of situations or events that are threats to health and organised on a population level by a societal action (such as a law or regulation) rather than at an individual level.

Health related quality of life: generally synonymous with "health status" but also encompassing reactions to coping with life circumstances.

Health services components

(1) Structure (of health systems or health facilities): aspects of the design of health services that influence the way in which services are delivered. These include the numbers and types of personnel and staff; the way in which these personnel are organised to do their work; the nature and extent of the facility and equipment; the range of services offered; systems of management and amenities; accessibility; mode of financing; the way in which the eligible population is determined and enumerated; and the mechanisms of governance and administrative decision making.

(2) Processes (of health care): aspects of the behaviour or performance of the health care system or health facility and the nature of participation on the part of people its serves. These consist of two parts: behaviours of the professionals and participation of the people who relate to the system or facility. Behaviours of professionals include the spectrum of clinical care involving problem (or needs) recognition, the diagnostic process, the recommendation of treatment or management; and appropriate follow up. Participation of people who relate to the system includes utilisation; understanding of recommendations, satisfaction with services rendered, and participation in decisions concerning strategies for management.

(3) Outcomes (of health care): those aspects of health that result from the interventions provided by the health system, the facilities and personnel that recommend them and the actions of those who are the targets of the interventions.

Health status: all aspects of physical and mental health and their manifestations in daily living, including impairment, disability, and handicap. Sometimes other aspects related to vulnerabilities and resiliencies are also included under this definition.

Prevention

Prevention: an intervention or interventions that interrupts the web of causality leading to one or more aspects of ill health.

Primary prevention: interruption of the chain of causality at a point or points before a physiological or psychological abnormality is identifiable.
Secondary prevention: interruption of the chain of causality at a point where physiological or psychological abnormality is present but before there is manifestation as a symptom or sign noticed by the individual.

Tertiary prevention: intervention after a sign or symptom is present, to reduce the likelihood of persistence or progression.

Primordial prevention: intervention at the most distal point in the chain of causality.

It is certain that there is a web of interactions that determine the state of health, from those that are most distal to those that are most proximal. The most distal are likely to be the political context in which the determinants are operative, followed by aspects of policy (health, social, environmental), followed by the context of social as well as medical contacts in communities, followed by individual social and behavioural characteristics (such as social isolation and health behaviours), followed by physiological states such as are related to perceived stressors. When interventions are designed to prevent the occurrence of a risk state, they are known as primary prevention. In a web of determinants, several interventions might be considered "primary". Primordial prevention occurs when there is a focus on a more antecedent primary preventive strategy. The most effective prevention focuses on the weakest part of the web and not necessarily on the most proximal (fig 1).

\section{Primary care}

Primary care: primary care is that aspect of a health services system that assures person focused care over time to a defined population, accessibility to facilitate receipt of care when it is first needed, comprehensiveness of care in the sense that only rare or unusual manifestations of ill health are referred elsewhere, and coordination of care such that all facets of care (wherever received) are integrated.

Funding: this work was supported in part by grant no 6U30CS00189-05 SI RI of the Bureau of Primary Health Care, Health Resources and Services Administration, Department of Health and Human Services, to the Primary Care Policy Center for the Underserved at Johns Hopkins University. 1 Stein REK, Westbook LE, Bauman LJ. The Questionnaire
for Identifying Children with Chronic Conditions
(QUICCC): A measure based on a non-categorical
approach. Pediatrics 1997;99:513-21.
2 Ottawa Charter for Health Promotion. First International
Conference on Health Promotion. Ottawa, Canada:
November 17-21, 1986.

General references

Burgess RG, ed. Key variables in social investigation. London: Routledge and Kegan Paul, 1986.

Last JM, ed. A dictionary of epidemiology. 3rd ed. New York: Oxford University Press, 1995. 